\title{
Singular Factorization of an Arbitrary Matrix
}

\author{
Gyan Bahadur Thapa ${ }^{1}$, P. Lam-Estrada ${ }^{2}$ J. López-Bonilla ${ }^{3 \text { a }}$ \\ ${ }^{1}$ Pulchowk Campus, Institute of Engineering, Tribhuvan University, Kathmandu, Nepal \\ ${ }^{2}$ ESFM-IPN, Depto. de Matemáticas, Edif. 9, Col. Lindavista, CP 07738, México DF \\ ${ }^{3}$ ESIME-Zacatenco, Instituto Politécnico Nacional (IPN), Edif. 5, CP 07738, México DF \\ ${ }^{a}$ Corresponding author: jlopezb@ipn.mx
}

Received: May 20, $2016 \quad$ Revised: July 25, $2016 \quad$ Accepted: July 28, 2016

\begin{abstract}
In this paper, we study the Singular Value Decomposition of an arbitrary matrix $\mathrm{A}_{n x m}$, especially its subspaces of activation, which leads in natural manner to the pseudo inverse of Moore -Bjenhammar - Penrose. Besides, we analyze the compatibility of linear systems and the uniqueness of the corresponding solution and our approach gives the Lanczos classification for these systems.
\end{abstract}

Keywords: SVD, Compatibility of linear systems, Pseudo inverse of a matrix

\section{Introduction}

For any real matrix $A_{n x m}$, Lanczos [18] constructs the matrix:

$$
S_{(n+m) x(n+m)}=\left(\begin{array}{cc}
0 & A \\
A^{T} & 0
\end{array}\right)
$$

and he studies the eigenvalue problem:

$$
S \vec{\omega}=\lambda \vec{\omega},
$$

where the proper values are real because $S$ is a real symmetric matrix. Besides,

$$
\text { rank } A \equiv p=\text { Number of positive eigenvalues of } S \text {, }
$$

such that $1 \leq p \leq \min (n, m)$. Then the singular values or canonical multipliers, thus called by Picard [26] and Sylvester [31], respectively, follow the scheme:

$$
\lambda_{1}, \lambda_{2}, \ldots, \lambda_{p},-\lambda_{1},-\lambda_{2}, \ldots,-\lambda_{p}, 0,0, \ldots, 0,
$$

that is, $\lambda=0$ has the multiplicity $n+m-2 p$. Only in the case $p=n=m$ can occur the absence of the null eigenvalue.

The proper vectors of $S$, named 'essential axes' by Lanczos, can be written in the form: 


$$
\vec{\omega}_{(n+m) x 1}=\left(\begin{array}{l}
\vec{u} \\
\vec{v}
\end{array}\right)_{m}^{n}
$$

then (1) and (2) imply the Modified Eigenvalue Problem:

$$
A_{n x m} \vec{v}_{m x 1}=\lambda \vec{u}_{n x 1}, \quad A_{m x n}^{T} \vec{u}_{n x 1}=\lambda \vec{v}_{m x 1},
$$

hence

$$
A^{T} A \vec{v}=\lambda^{2} \vec{v}, \quad A A^{T} \vec{u}=\lambda^{2} \vec{u},
$$

with special interest in the associated vectors with the positive eigenvalues because they permit to introduce the matrices:

$$
U_{n x p}=\left(\vec{u}_{1}, \vec{u}_{2}, \ldots, \vec{u}_{p}\right), \quad V_{m x p}=\left(\vec{v}_{1}, \vec{v}_{2}, \ldots, \vec{v}_{p}\right),
$$

verifying $U^{T} U=V^{T} V=I_{p x p}$ because:

$$
\vec{u}_{j} \cdot \vec{u}_{k}=\vec{v}_{j} \cdot \vec{v}_{k}=\delta_{j k},
$$

therefore $\vec{\omega}_{j} \cdot \vec{\omega}_{k}=2 \delta_{j k}, j, k=1,2, \ldots, p$. Thus, the Singular Value Decomposition (SVD) express that $A$ is the product of three matrices [18 - 21]:

$$
A_{n x m}=U_{n x p} \Lambda_{p x p} V_{p x m}^{T}, \quad \Lambda=\operatorname{Diag}\left(\lambda_{1}, \lambda_{2}, \ldots, \lambda_{p}\right) .
$$

This relation tells that in the construction of $A$ we do not need information about the null proper value; the information from $\lambda=0$ is important to study the existence and uniqueness of the solutions for a linear system associated to $A$. This approach of Lanczos is similar to the methods in $[15,16,27,28]$. It can be considered that Jordan [15, 16], Sylvester [30, 31] and Beltrami [2] are the founders of the SVD [29], and there is abundant literature [4, 6, 7, 11, 30, 34] on this matrix factorization and its applications.

The rest of the paper is planned as follows: In Section 2, we realize an analysis of the proper vectors $\vec{\omega}_{j}, j=1, \ldots, n+m$, associated to the eigenvalues (4), which leads to the subspaces of activation of $A$ with the pseudo inverse of Moore [22], Bjerhammar [3] and Penrose [25]. In Section 3, we study the compatibility of linear systems, with special emphasis in the important participation of the null singular value and its corresponding eigenvectors. Finally, Section 4 concludes the paper.

\section{Subspaces of Activation and Natural Inverse Matrix}

From (6), the proper vectors associated with the positive eigenvalues verify:

$$
A \vec{v}_{j}=\lambda_{j} \vec{u}_{j}, \quad A^{T} \vec{u}_{j}=\lambda_{j} \vec{v}_{j}, \quad j=1, \ldots, p
$$

then 


$$
A\left(-\vec{v}_{j}\right)=\left(-\lambda_{j}\right) \vec{u}_{j}, \quad A^{T} \vec{u}_{j}=\left(-\lambda_{j}\right)\left(-\vec{v}_{j}\right),
$$

that is,

$$
S\left(\begin{array}{c}
\vec{u}_{k} \\
\vec{v}_{k}
\end{array}\right)=\lambda_{k}\left(\begin{array}{c}
\vec{u}_{k} \\
\vec{v}_{k}
\end{array}\right) \quad \text { implies } S\left(\begin{array}{c}
\vec{u}_{k} \\
-\vec{v}_{k}
\end{array}\right)=\left(-\lambda_{k}\right)\left(\begin{array}{c}
\vec{u}_{k} \\
\vec{v}_{k}
\end{array}\right),
$$

therefore, the eigenvectors $\left(\begin{array}{c}\vec{u}_{j} \\ \vec{v}_{j}\end{array}\right)$ and $\left(\begin{array}{c}\vec{u}_{j} \\ -\vec{v}_{j}\end{array}\right)$ correspond to the proper values $\lambda_{1}, \ldots, \lambda_{p}$ and $-\lambda_{1}, \ldots,-\lambda_{p}$, respectively. Thus we must have $n+m-2 p$ eigenvectors connected to $\lambda=0$, which is denoted by $\vec{\omega}_{r}^{(0)}$, and from (6) we further have:

$$
\begin{gathered}
\vec{\omega}_{j}^{(0)}=\left(\begin{array}{c}
\vec{u}_{j}^{(0)} \\
0 \\
\vdots \\
0
\end{array}\right)_{m}^{n}, \quad A^{T} \vec{u}_{j}^{(0)}=\overrightarrow{0}, \quad j=1, \ldots, n-p, \\
\vec{\omega}_{(n-p)+k}^{(0)}=\left(\begin{array}{c}
0 \\
\vdots \\
0 \\
\vec{v}_{k}^{(0)}
\end{array}\right)_{m}^{n}, \quad A \vec{v}_{k}^{(0)}=\overrightarrow{0}, \quad k=1, \ldots, m-p .
\end{gathered}
$$

The conditions (14) and (15) can be multiplied by $A$ and $A^{T}$, then $\vec{u}_{j}^{(0)}$ and $\vec{v}_{k}^{(0)}$ are eigenvectors of the Gram matrices $A A^{T}$ and $A^{T} A$ :

$$
\left(A A^{T}\right)_{n x n} \vec{u}_{j}^{(0)}=\overrightarrow{0}, \quad\left(A^{T} A\right)_{m x m} \vec{v}_{k}^{(0)}=\overrightarrow{0}
$$

but by (7) these matrices have $p$ proper vectors for $\lambda_{1}, \ldots, \lambda_{p}$, therefore only there are $n-$ $p$ and $m-p$ vectors $\vec{u}_{j}^{(0)}$ and $\vec{v}_{k}^{(0)}$, that can be selected with orthonormality:

$$
\vec{u}_{j}^{(0)} \cdot \vec{u}_{r}^{(0)}=\delta_{j r}, \quad \vec{v}_{k}^{(0)} \cdot \vec{v}_{q}^{(0)}=\delta_{k q}
$$

that is, $\vec{\omega}_{j}^{(0)} \cdot \vec{\omega}_{k}^{(0)}=\delta_{j k}$, then $\left\{\vec{u}_{j}^{(0)}\right\}$ and $\left\{\vec{v}_{k}^{(0)}\right\}$ are bases for the Kernel $A^{T}$ and Kernel $A$, respectively.

If we employ (10) in (14), SVD of $A$ results $V \Lambda U^{T} \vec{u}_{j}^{(0)}=\overrightarrow{0}$, whose multiplication by the left with $\Lambda^{-1} V^{T}$ [remembering that $U^{T} U=V^{T} V=I$ ], gives the compatibility condition:

$$
U^{T} \vec{u}_{j}^{(0)}=\overrightarrow{0} \quad \Rightarrow \quad \vec{u}_{r} \cdot \vec{u}_{j}^{(0)}=0, \quad r=1, \ldots, p ; \quad j=1, \ldots, n-p,
$$

equivalently

$$
\operatorname{Col} U \perp \vec{u}_{k}^{(0)}, \quad k=1, \ldots, n-p .
$$

Similarly, if we use SVD into (15) and we multiply by $\Lambda^{-1} U^{T}$ : 
80 Singular Factorization of an Arbitrary Matrix

$$
\begin{aligned}
& V^{T} \vec{v}_{k}^{(0)}=\overrightarrow{0}, \quad \vec{v}_{r} \cdot \vec{v}_{k}^{(0)}=0, \quad r=1, \ldots, p ; \quad k=1, \ldots m-p \\
& \therefore \quad \text { Col } V \perp \vec{v}_{j}^{(0)}, \quad j=1, \ldots, m-p .
\end{aligned}
$$

It is convenient to make two remarks:

Remark 1: From $A=U \Lambda V^{T}$ is evident that the matrices $U, \Lambda$ and $V$ permit to construct $A$, but is useful to know more about the structure of $A$ and its transpose:

$$
A=\left(\begin{array}{lll}
\vec{a}_{1} \ldots \vec{a}_{m}
\end{array}\right), \quad A^{T}=\left(\begin{array}{lll}
\vec{c}_{1} & \ldots & \vec{c}_{n}
\end{array}\right),
$$

where $\left(\vec{a}_{j}\right)_{n x 1}$ and $\left(\vec{c}_{k}\right)_{m x 1}$ are the corresponding columns. Then from (10) we obtain the expressions:

$$
\vec{a}_{j}=\lambda_{1} v_{1}^{(j)} \vec{u}_{1}+\cdots+\lambda_{p} v_{p}^{(j)} \vec{u}_{p}, \quad j=1, \ldots m, \quad \vec{c}_{k}=\lambda_{1} u_{1}^{(k)} \vec{v}_{1}+\cdots+\lambda_{p} u_{p}^{(k)} \vec{v}_{p}, \quad k=1, \ldots, n
$$

with the notation:

$$
v_{r}^{(j)}=j \text { th }- \text { component of } \vec{v}_{r}
$$

and similar for $u_{r}^{(k)}$; we observe that $\vec{c}_{k}^{T}$ are the rows of $A$.

From (23) are immediate the equalities of subspaces:

$$
\operatorname{Col} A=\operatorname{Col} U, \quad \operatorname{Row} A=\operatorname{Col} V,
$$

but $\operatorname{dim} \operatorname{Col} U=\operatorname{dim} \operatorname{Col} V=p$, then:

$$
\operatorname{rank} A=\operatorname{dim} \operatorname{Col} A=\operatorname{dim} \operatorname{Row} A=p
$$

in according with (3).

Remark 2: We have the rank-nullity theorem [24, 32, 33]:

$$
\operatorname{dim}(\text { Kernel } A)+\operatorname{rank} A=m,
$$

therefore $\operatorname{dim}($ Kernel $A)=m-p$, by this reason there are $(m-p)$ vectors $\vec{v}_{k}^{(0)}$ with the property (15). Besides,

$$
\operatorname{dim}\left(\operatorname{Kernel} A^{T}\right)+\operatorname{rank} A^{T}=n,
$$

but $\operatorname{rank} A^{T}=\operatorname{rank} A=p$, then $\operatorname{dim}\left(\right.$ Kernel $\left.A^{T}\right)=n-p$ in harmony with the $(n-$ $p$ ) vectors $\vec{u}_{j}^{(0)}$ verifying (14).

If $A_{n x m}$ acts on an arbitrary vector $\vec{x} \varepsilon E_{m}$ produces a vector $\vec{y} \varepsilon E_{n}$, with the decompositions: 


$$
\vec{x}=\vec{x}^{(0)}+\vec{x}_{\mathrm{CV}}, \quad \vec{y}=\vec{y}^{(0)}+\vec{y}_{\mathrm{CU}},
$$

where

$$
\begin{aligned}
& \vec{x}^{(0)} \varepsilon \operatorname{Kernel} A, \quad \vec{x}_{\mathrm{CV}} \varepsilon \operatorname{Col} V, A \vec{x}^{(0)}=\overrightarrow{0}, \quad \vec{x}^{(0)} \cdot \vec{x}_{\mathrm{CV}}=0, \\
& \vec{y}^{(0)} \varepsilon \operatorname{Kernel} A^{T}, \quad \vec{y}_{\mathrm{CU}} \varepsilon \operatorname{Col} U, \quad A^{T} \vec{y}^{(0)}=\overrightarrow{0}, \quad \vec{y}^{(0)} \cdot \vec{y}_{\mathrm{CU}}=0,
\end{aligned}
$$

thus we say that $A$ is activated into the subspaces $\operatorname{Col} U$ and $\operatorname{Col} V$.

Therefore, $A \vec{x}=A \vec{x}_{\mathrm{CV}}=\vec{y}$ and in the construction of $\vec{y}$ we lost the information about $\vec{x}^{(0)}$, then it is not possible to recover $\vec{x}$ from $\vec{y}$, that is, it is utopian to search for an 'inverse matrix' acting on $\vec{y}$ to give $\vec{x}$. However, when $\vec{x}^{(0)}=\overrightarrow{0}$ and $\vec{y}^{(0)}=\overrightarrow{0}$ we can introduce a 'natural inverse matrix', thus named it by Lanczos, which coincides with the pseudo inverse of Moore [22], Bjerhammar [3] and Penrose [25]:

"Any matrix $A_{n x m}$, restricted to its subspaces of activation, always can be inverted".

In fact, if $\vec{x} \varepsilon \operatorname{Col} V$ is an arbitrary vector, $\vec{x}=q_{1} \vec{v}_{1}+\cdots+q_{p} \vec{v}_{p}$, then from (6):

$$
A \vec{x}=\lambda_{1} q_{1} \vec{u}_{1}+\cdots+\lambda_{p} q_{p} \vec{u}_{p}=\vec{y} \varepsilon \operatorname{Col} U,
$$

and now we search the inverse natural $A_{N \text { mxn }}^{-1}$ such that:

$$
A_{N}^{-1} \vec{y}=\vec{x}
$$

or more general:

$$
A_{N}^{-1} A \vec{x}=\vec{x}, \quad \forall \vec{x} \varepsilon \operatorname{Col} V, \quad A A_{N}^{-1} \vec{y}=\vec{y}, \quad \forall \vec{y} \varepsilon \operatorname{Col} U .
$$

If the decomposition (10) is applied to (32), we deduce the natural inverse matrix:

$$
A_{N \text { mxn }}^{-1}=V_{m x p} \Lambda_{p x p}^{-1} U_{p x n}^{T}
$$

satisfying (33) and (34). With (35), it is easy to prove the properties [24, 32]:

$$
A A_{N}^{-1} A=A, \quad A_{N}^{-1} A A_{N}^{-1}=A_{N}^{-1}, \quad\left(A A_{N}^{-1}\right)^{T}=A A_{N}^{-1}, \quad\left(A_{N}^{-1} A\right)^{T}=A_{N}^{-1} A,
$$

which characterize the pseudo inverse of Moore - Bjerhammar - Penrose, that is, the inverse matrix $[8,9,12]$ of these authors coincides with the natural inverse (35) deduced by Lanczos [18 $-21]$.

In the SVD only participate the positive proper values of $S$, without the explicit presence of the vectors $\vec{u}_{j}^{(0)}$ and $\vec{v}_{k}^{(0)}$ associated with the null eigenvalue, then it is natural to investigate the role performed by the information related with $\lambda=0$. In Section 3, we study linear systems where $A$ is the corresponding matrix of coefficients, and we exhibit that the $\vec{u}_{j}^{(0)}$ permit to 
analyze the compatibility of such systems; besides, when they are compatibles then with the $\vec{v}_{k}^{(0)}$, we search if the solution is unique. In other words, the null eigenvalue does not participates when we consider to $A$ as an algebraic operator and we construct its factorization (10), but $\lambda=0$ is important if $A$ acts as the matrix of coefficients of a linear system.

\section{Compatibility of Linear Systems}

A Linear System of $n$ equations with $m$ unknowns can be written in the matrix form:

$$
A_{n x m} \vec{x}_{m x 1}=\vec{b}_{n x 1}
$$

where (10) implies that $U \Lambda V^{T} \vec{x}=\vec{b}$ whose multiplication by $\vec{u}_{j}^{(0) T}$ gives the compatibility conditions:

$$
\vec{u}_{j}^{(0)} \cdot \vec{b}=0, \quad j=1, \ldots, n-p
$$

due to (19). Then the system (37) is compatible if $\vec{b}$ is orthogonal to all independent solutions of the adjoint system $A^{T} \vec{u}=\overrightarrow{0}$, therefore:

$$
\text { "A } \vec{x}=\vec{b} \text { has solution if } \vec{b} \varepsilon \operatorname{Col} U ",
$$

which is the traditional formulation [6] of the compatibility condition for a given linear system. From (25) and (39) is clear that $A$ and the augmented matrix $(A \vec{b})$ have the same column space:

$$
\operatorname{Col} A=\operatorname{Col}(A \vec{b})=\operatorname{Col} U,
$$

thus at the books [32] we find the result:

$$
\text { " } A \vec{x}=\vec{b} \text { is compatible if } \operatorname{rank} A=\operatorname{rank}(A \vec{b}) " .
$$

If $\vec{b} \varepsilon \operatorname{Col} U$, then from (11):

$$
\vec{b}=b^{(1)} \vec{u}_{1}+\cdots+b^{(p)} \vec{u}_{p}=A \vec{Q}, \quad \vec{Q}=\frac{b^{(1)}}{\lambda_{1}} \vec{v}_{1}+\cdots+\frac{b^{(p)}}{\lambda_{p}} \vec{v}_{p},
$$

and (37) leads to:

$$
A(\vec{x}-\vec{Q})=\overrightarrow{0}
$$

The set of solutions of (43) is the Kernel $A$ with dimension $(m-p)$ due to (27), therefore (43) has the unique solution $\vec{x}-\vec{Q}=\overrightarrow{0}$ when $p=m$, that is, when rank $A$ coincides with the number of unknowns we have not vectors $\vec{v}_{k}^{(0)} \neq \overrightarrow{0}$ verifying $A \vec{v}_{k}^{(0)}=\overrightarrow{0}$. Then:

"The compatible system $A \vec{x}=\vec{b}$ has unique solution only when $p=m$ ",

besides from (24) and (42) we obtain that $b^{(k)}=\vec{b} \cdot \vec{u}_{k}, \vec{x}=\vec{Q}$ and: 


$$
x_{r}=Q^{(r)}=\frac{b^{(1)}}{\lambda_{1}} v_{1}^{(r)}+\cdots+\frac{b^{(p)}}{\lambda_{p}} v_{p}^{(r)}=\vec{b} \cdot \vec{t}_{r}, \quad r=1, \ldots, m
$$

where

$$
\vec{t}_{r}=\frac{v_{1}^{(r)}}{\lambda_{1}} \vec{u}_{1}+\cdots+\frac{v_{p}^{(r)}}{\lambda_{p}} \vec{u}_{p} \quad \varepsilon \operatorname{Col} U
$$

thus the value of each unknown is the projection of $\vec{b}$ onto each vector (46). In consequence, $\vec{b} \varepsilon \operatorname{Col} U$ guarantees the solution of (37), and it is unique only if $p=m$.

Besides, from (42) we see that the solution $\vec{x}=\vec{Q}$ implies that $\vec{x} \varepsilon \operatorname{Col} V$, then we have system $\quad A \vec{x}=\vec{b}$ where $\vec{x}$ and $\vec{b}$ are totally embedded into $\operatorname{Col} V$ and $\operatorname{Col} U$, respectively, that is, $\vec{x}$ and $\vec{b}$ are into the subspaces of activation of $A$, thus from (32) and (33) there is the natural inverse $A_{N}^{-1}$ such that:

$$
\begin{aligned}
& \vec{x}=A_{N}^{-1} \vec{b}=V_{m x m} \Lambda_{m x m}^{-1} U_{m x n}^{T} \vec{b}=V \Lambda^{-1}\left(\begin{array}{c}
b^{(1)} \\
\vdots \\
b^{(m)}
\end{array}\right)=V\left(\begin{array}{c}
\frac{b^{(1)}}{\lambda_{1}} \\
\vdots \\
\frac{b^{(m)}}{\lambda_{m}}
\end{array}\right)= \\
& \left(\begin{array}{c}
\frac{b^{(1)}}{\lambda_{1}} v_{1}^{(1)}+\cdots+\frac{b^{(m)}}{\lambda_{m}} v_{m}^{(1)} \\
\vdots \\
\frac{b^{(1)}}{\lambda_{1}} v_{1}^{(m)}+\cdots+\frac{b^{(m)}}{\lambda_{m}} v_{m}^{(m)}
\end{array}\right), \quad p=m,
\end{aligned}
$$

in according with (45). The vectors (46) are important because their inner products with $\vec{b}$ give the solution of (37) via (45), and they also are remarkable because permit to construct the natural inverse:

$$
A_{N}^{-1}{ }_{m x n}=\left(\vec{t}_{1} \vec{t}_{2} \ldots \vec{t}_{m}\right)^{T}, \quad p=m .
$$

Lanczos [6] considers three situations:

i) $\quad n<m$ : The linear system is under-determined because it has more unknowns than equations, and from $1 \leq p \leq \min (n, m)$ is impossible the case $p=m$, therefore, if (37) is compatible then its solution cannot be unique.

ii) $\quad n=m$ : The system is even-determined with unique solution when $p=m$, that is, if $\operatorname{det} A \neq 0$. In this case also $p=n$, we have not vectors $\vec{u}_{j}^{(0)} \neq \overrightarrow{0}$, thus $\vec{b} \varepsilon \operatorname{Col} U$ and automatically the system is compatible.

iii) $\quad n>m$ : The linear system is over-determined, and by $1 \leq p \leq \min (n, m)$ can occur the case $p=m$ for unique solution if the system is compatible.

Hence it is immediate the classification of linear systems introduced by Lanczos [21]:

Free and complete: $\quad p=n=m$, unique solution,

Restricted and complete: 


$$
p=m<n \text {, over-determined, unique solution, }
$$

Free and incomplete: $\quad p=n<m$, under-determined, non-unique solution,

Restricted and incomplete: $p<n$ and $p<m$, solution without uniqueness,

with the meaning:

Free: The conditions (30) are satisfied trivially.

Restricted: It is necessary to verify that $\vec{b} \varepsilon \operatorname{Col} U$.

Complete: The solution has uniqueness.

Incomplete: Non-unique solution.

When $p \neq m$, the homogeneous system $A \vec{v}=\overrightarrow{0}$ has the non-trivial solutions $\vec{v}_{k}^{(0)}$, then from (27) we conclude that the general solution of (37) is:

$$
\vec{x}=\vec{Q}+c_{1} \vec{v}_{1}^{(0)}+\cdots+c_{m-p} \vec{v}_{m-p}^{(0)},
$$

where the $c_{k}$ are arbitrary constants.

\section{Conclusion}

With the SVD we can find the subspaces of activation of $A$, and it leads to the natural inverse [6, 26-28] of any matrix, known it in the literature as the Moore-Penrose pseudo inverse. Besides, the SVD gives a better understanding of the compatibility of linear systems. On the other hand, Lanczos [21] showed that the Singular Value Decomposition provides a universal platform to study linear differential and integral operators for arbitrary boundary conditions. We note that the term 'singular value' was introduced by Green [10] (see [5] too) in his studies on electromagnetism. The SVD is very useful to study the rotation matrix in classical mechanics [14] and to comprehend the matrix technique to deduce gauge transformations of Lagrangians [17]. For a graphic example of the use of the SVD in image processing, we refer see [1]; and for its use in cryptography, we refer [23]. Heat [13] mentions software for singular value computations.

\section{References}

[1] Andrews HC and Patterson CL (1975), Outer product expansions and their uses in digital image processing. Am. Math. Monthly, 82: 1-13.

[2] Beltrami E (1873), Sulle funzioni bilineari. Giornale di Mathematische, 11: 98-106.

[3] Bjerhammar A (1951), Application of calculus of matrices to method of least squares, with special references to geodetic calculations. Trans. Roy. Inst. Tech. Stockholm, (49): 1-86.

[4] Blank SJ, Krikorian N and Spring D (1989), A geometrically inspired proof of the singular value decomposition. Am. Math. Monthly 96(3): 238-239. 
[5] Cannell DM (2001), George Green, mathematician \& physicist 1793-1841. SIAM, Philadelphia, USA.

[6] Eckart C and Young G (1939), A principal axis transformation for non-hermitian matrices. Bull. Amer. Math. Soc., 45(2) : 118-121.

[7] Gaftoi V, López-Bonilla J and Ovando G (2007), Singular value decomposition and Lanczos potential, in "Current topics in quantum field theory research", Ed. O. Kovras, Nova Science Pub., New York, Chap. 10, 313-316.

[8] Golub GH and Kahan W (1965), Calculating the singular values and pseudo inverse of a matrix. SIAM J. Numer.Anal. B2: 205-224.

[9] Greville TNE (1960), Some applications of the pseudo inverse of a matrix. SIAM Rev., 2 (1) : 15-22.

[10] Green G (1828), An essay on the application of mathematical analysis to the theories of electricity and magnetism. Private subscription, only fifty copies, reprinted in three parts at $J$. Reine Angewand.Math. 39 (1850) 73-89, 44 (1852) 356-374 and 47 (1854): 161-212.

[11] Guerrero IM, López-Bonilla J and Rosales RL (2012), SVD applied to Dirac super matrix, The SciTech, J. Sci. \& Tech. Special Issue, Aug. 111-114.

[12] Hazra AK (2006), Matrix algebra, calculus and generalized inverse. Cambridge Int. Sci. Pub.

[13] Heat MT (1997), Scientific computing: An introductory survey. Chap. 4, McGraw-Hill, New York.

[14] Hernández GA, López-Bonilla J and Rivera RJ (2012), A similarity transformation for the rotation matrix. Int. J. Math. Eng. Sci., 1(1): 8-12.

[15] Jordan C (1874), Mémoire sur les forms bilinéaires. J. de Mathématiques Pures et Appliquées, Deuxieme Série, 19: 35-54.

[16] Jordan C (1874), Sur la réduction des formes bilinéaires. Compt. Rend. Acad. Sci. Paris, 78: $614-617$.

[17] Lam P, López-Bonilla J, López-Vázquez R and Ovando G (2015), Matrix method to construct point symmetries of Lagrangians, Bull. of Kerala Mathematics Association, 12(1): 43-52.

[18] Lanczos C (1958), Linear systems in self-adjoint form. Am. Math. Monthly, 65(9): 665 - 679.

[19] Lanczos C (1960), Extended boundary value problems. Proc. Int. Congr. Math. Edinburgh1958, Cambridge University Press, 154-181.

[20] Lanczos C (1966), Boundary value problems and orthogonal expansions. SIAM J. Appl. Math., 14(4): $831-863$

[21] Lanczos C (1997), Linear Differential Operators, Dover, New York.

[22] Moore EH (1920), On the reciprocal of the general algebraic matrix. Bull. Am. Math. Soc., 26 (9) : 394-395.

[23] Moler CB and Morrison D (1983), Singular value analysis of cryptograms. Am. Math. Monthly, 90 : 78-87.

[24] Nakos G and Joyner D (1998), Linear algebra with applications. Chap. 8, Brooks/Cole Pub. Co, New York.

[25] Penrose R (1955), A generalized inverse for matrices. Proc. Camb. Phil. Soc., 51: 406-413. 
[26] Picard E (1910), Sur un theorem general relative aux integrals de premier espéce et sur quelques problémes de physique mathématique. Rend. Circ. Mat. Palermo, 25: 79-97.

[27] Schmidt E (1907), Zur theorie der linearen und nichtlinearen integralgleichungen. Teil 1, Mathematische Annalen Bd. 63: 433-476 and 64: 161-174.

[28] Smithies F (1963), Linear differential operators. The Mathematical Gazette 47: 265-266.

[29] Stewart GW (1993), On the early history of the SVD. SIAM Review, 35: 551-566

[30] Sylvester JJ (1889), Sur la réduction biorthogonale d'une forme linéo-linéaire á sa forme cannonique. Compt. Rend. Acad. Sci. Paris, 108: 651-653.

[31] Sylvester JJ (1889), On the reduction of a bilinear quantic of the nth order to the form of a sum of $\mathrm{n}$ products. Messenger of Mathematics, 19: 42-46.

[32] Weyr E (1885), Répartition des matrices en espaces et formation de toutes les espaces. $C . R$. Acad. Sci. Paris,100: 966-969.

[33] Weyr E (1890), Zur theorie der bilinearen formen. Mon. für Mathematik und Physik, 1 : 163236.

[34] Yanai H, Takeuchi K and Takane Y (2011), Projection matrices, generalized inverse matrices, and singular value decomposition. Chap. 3, Springer, New York. 